\title{
1 Scale-similarity model for Lagrangian velocity correlations in isotropic and stationary turbulence
}

2

3

5

6

7

8

9

10

11

12

13

AQ: 18 The turbulent diffusion process is naturally described by \#1 19 the relative separations of particle pairs in Lagrangian coor20 dinates [1-3]. Following Taylor's pioneering work [4], 21 Batchelor expressed the root-mean-square of the relative 22 separations by the integration of Lagrangian velocity corre23 lation (LVC) [5,6]. Therefore, an accurate prediction on rela24 tive dispersions is essentially dependent on LVC modeling. 25 In the probability density function (PDF) approach [7-9] and 26 various turbulence closure theories $[2,10]$, the LVC provides 27 a relaxation time scale as an input to close the transport 28 equations. A related question is the relationship between La29 grangian and Eulerian velocity correlations [11-13], which 30 yields the different predictions on the scaling of energy spec31 tra. The recent application of large-eddy simulation (LES) to 32 particle-laden turbulence raises such a question as whether or 33 not LES could correctly predict LVCs [14]. The existing sub34 grid scale models (SGS) are mostly constructed to predict the 35 spatial statistics such as energy spectra $[15,16]$. However, 36 these models may not accurately predict LVCs since the 37 LVCs are not dependent on energy spectra alone. A LVC 38 model is helpful to develop a SGS model for LES of particle39 laden turbulence. In this paper, we will develop a scale40 similarity model for Lagrangian velocity correlations.

41 The inherent difficulty in developing a LVC model is that 42 a LVC depends on its instantaneous space separations. 43 Therefore, the LVC and the relative separation are strongly 44 correlated and thus their relationship forms an unclosed 45 equation. This implies that the possession of such a relation46 ship would be equivalent to solve a turbulence closure prob47 lem. Starting with the Taylor frozen flow hypothesis, Smith 48 and Hay [17] developed a model which expresses the LVC 49 by an Eulerian velocity correlation and a linear transforma50 tion of space and time separations. We will show in next 51 section that this model is a first approximation to the isocor-

\footnotetext{
*Author to whom all correspondence should be addressed; hgw@1nm.imech.ac.cn; guoweihe@yahoo.com
}

relation contours. Some corrections [18] to the Smith-Hay 52 model were made to include the nonfrozen flow effect with 53 some successes. The critical assumption in those corrections 54 is that the LVCs have the same shape as the Eulerian velocity $\mathbf{5 5}$ correlations with different decorrelation time scales. Taylor 56 assumed that the space and time variables in the LVC are 57 separable [6]. This implies that the LVC is a simple product 58 of Eulerian velocity correlations and one-point LVC. This 59 assumption suppresses the space-scale-dependence of the 60 temporal part of LVCs.

Since both Lagrangian and Eulerian velocity correlations 62 are determined by the same turbulent flows, there must exist 63 a relationship between those two quantities. To understand 64 this issue, we define a two-point and two-time Lagrangian 65 velocity correlation or simply Lagrangian velocity correla- 66 tion as follows:

$$
R(r, \tau)=\left\langle\mathbf{v}\left(\mathbf{x}_{0}+\mathbf{r}, t_{0} \mid t_{0}+\tau\right) \cdot \mathbf{v}\left(\mathbf{x}_{0}, t_{0} \mid t_{0}\right)\right\rangle,
$$

where $\mathbf{v}\left(\mathbf{x}, t_{0} \mid t_{0}+\tau\right)$ is the velocity, measured at time $t_{0}+\tau$, of 69 the fluid particle that passes through the point $\mathbf{x}$ at time $t_{0} . \tau 70$ is a time separation which is either positive for a forward 71 trace or negative for a backward trace. $\mathbf{r}$ denotes a space 72 separation vector with its magnitude $r=|\mathbf{r}|$ and $-\mathbf{r}$ denotes 73 the space separation vector of the separation $-r=-|\mathbf{r}|$. The $\mathbf{7 4}$ bracket $\langle\cdots\rangle$ denotes the ensemble average. The correlation 75 function is independent of the spatial location $\mathbf{x}_{0}$ and the 76 starting time $t_{0}$ due to the homogeneity and stationarity. 77 When $r=0$, the LVC $R(r, \tau)=R(0, \tau)$ is the Lagrangian one- 78 point, two-time correlation. When $\tau=0$, the LVC $R(r, \tau) 79$ $=R(r, 0)$ coincides with the conventional two-point Eulerian 80 velocity correlation. Kolmogrov's similarity hypothesis [19] 81 yields a self-similar function for either space correlation $\mathbf{8 2}$ $R(r, 0)$ or time correlation $R(0, \tau)$. However, the space-time 83 correlation function $R(r, \tau)$ remains unknown [20].

To pursue this relationship, the two-point, one-time LVC 85 is expressed in terms of the joint PDF of the relative separa- 86 tion and Eulerian velocity. Corrsin [21] proposed the well- 87 known independent hypothesis that the PDF of relative sepa- 88 rations are independent of that of the Eulerian velocity fields. 89 The hypothesis circumvents the main difficulty in relating 90 
91 Lagrangian velocity correlations with Eulerian ones and 92 yields an approximate relationship between the Lagrangian 93 and Eulerian velocity correlations. Latter on, this result is 94 shown to be equivalent to the first-order truncation of renor95 malized perturbation expansion [22]. Among the statistic 96 theories of turbulence, direct interaction approximation 97 (DIA) [23] and Lagrangian renormalization approximation 98 (LRA) [24] are typically used to calculate the LVCs. They 99 successfully predict the scaling of LVCs in the inertial sub100 range with good agreements with the DNS results. However, 101 no explicit model can be analytically deduced from either of 102 the DIA and LRA equations. Gotoh and Kaneda [25] used 103 the Taylor series expansion to calculate the Lagrangian time 104 scales. The short-time analysis also yields a good prediction 105 on the scaling of Lagrangian time scales. The Langevin 106 equations can be also used to calculate LVCs [2,26-28]. Re107 cent experimental measurements [29] on pair dispersion in 108 turbulent flows attract the attention to Lagrangian statistics 109 and raise such a problem of the intermittency correction to 110 Lagrangian time scales.

111 In this paper, we will develop a scale-similarity model for 112 LVC using the isocorrelation contours, which is related to the 113 Eulerian velocity correlation with an additional parameter. 114 The isocorrelation contours represent a similarity transfor115 mation which links the properties of the LVC at different 116 scales in space and time. Once the transformation is applied 117 to link the two-point, two-time LVCs with the two-point, 118 one-time LVCs, it yields a scale-similarity model.

119 The organization of this paper is as follows: we will de120 rive the scale-similarity model in Sec. II. Unlike the Smith121 Hay model, the present model doesn't depend on the Taylor 122 frozen flow hypothesis. It is a second approximation to the 123 isocorrelation contours while the Smith-Hay model is only a 124 first approximation. Using the Kolmogorov similarity hy125 pothesis, this model can be generalized to the space and time 126 separations in the inertial range. It shares the short-time be127 haviors with the results from Taylor series expansions, but it 128 is valid for larger separations in both space and time. We 129 analytically derive the expression for the dispersion velocity, 130 which is the only one parameter in the scale-similarity model 131 in Sec. III This derivation is based on the conventional qua132 sinormality assumption. From the expression of the disper133 sion velocity, we confirm the intuitive prediction that the 134 Lagrangian decorrelation time scale is larger than the Eule135 rian one. The direct numerical simulation (DNS) of isotropic 136 turbulence will be used to verify the scale-similarity model 137 in Sec. IV The data from DNS supports the scale-similarity 138 model in the sense of that the correlation curves collapse 139 together when they are rescaled using the present model. 140 Finally, the discussion and conclusions are made in Sec. V

\section{SCALE-SIMILARITY MODEL}

$A Q: 142$ In this section, we will first present the scale-similarity \#3 143 model for Lagrangian velocity correlations and then present 144 its derivation. The scale-similarity model expresses a two145 point and two-time LVC $R(r, \tau)$ by the Eulerian velocity cor146 relation $R(r, 0)$ and a parameter $V$, namely,

$$
R(r, \tau)=R\left(\sqrt{r^{2}+V^{2} \tau^{2}}, 0\right) .
$$

Here, $V$ is a dispersion velocity which will be explained later. 148 The model incorporates the time separation into the argu- 149 ment of the space separation in the Eulerian velocity corre- 150 lations. The well-known Smith-Hay model suggests

$$
R(r, \tau)=R(r+U \tau / \beta, 0),
$$

where $U$ is a sweeping velocity defined as root-mean-square 153 of fluctuation velocities and $\beta$ a constant ratio of Lagrangian 154 to Eulerian integral time scales. The Smith-Hay model and 155 its variations imply that the isocorrelation contours are 156 straight lines. This implication violates the property of cor- 157 relation functions which should decay as either space or time 158 separation increases negatively. The scale-similarity model 159 does exhibit this property.

160

The present model is based on the scale-similarity behav- 161 ior of the LVCs, which is the consequence of the Kolmog- 162 orov similarity hypothesis. Figure 2 shows the isocorrelation 163 contours from the DNS data of isotropic turbulence, whose 164 details will be given in Sec. IV It is clearly seen that they are 165 closed curves of maximal chord length in the space separa- 166 tion axis and minimal chord length in the time separation 167 axis. The overall shapes of the isocorrelation contours are 168 mainly determined by the aspect ratio that is the ratio of the 169 maximal chord length to the minimal chord one. An empiri- 170 cal observation suggests that the isocorrelation contours are 171 self-similar in the sense of that their aspect ratios satisfy a 172 power law. This can be justified from the classic Kolmog- 173 orov similarity hypothesis as follows: we introduce the 174 second-order Lagrangian velocity structure function in both 175 space and time

176

$$
D(r, \tau)=\left\langle\left[v_{i}(\mathbf{x}+\mathbf{r}, t \mid t+\tau)-v_{i}(\mathbf{x}, t \mid t)\right]^{2}\right\rangle .
$$

It is easily found that $D(r, 0)$ is a Eulerian velocity structure 178 function in space and $D(0, \tau)$ a Lagrangian velocity structure 179 function in time. The Lagrangian velocity structure functions 180 are related to the LVCs by

$$
2 R(r, \tau)=2 R(0,0)-D(r, \tau) .
$$

We present $r_{c}$ as the intersecting point of the contour 183 $R(r, \tau)=C$ with the space separation axis and $\tau_{c}$ as the inter- 184 secting one of the contours $R(r, \tau)=C$ with the time separa- 185 tion axis. The parameter $C$ is a contour level, such that 186

$$
R\left(r_{c}, 0\right)=R\left(0, \tau_{c}\right)=C,
$$

which defines the aspect ratio $r_{c} / \tau_{c}$. Noting Eq. (5), Eq. (6) 188 implies

$$
D\left(r_{c}, 0\right)=D\left(0, \tau_{c}\right) .
$$

If both $r_{c}$ and $\tau_{c}$ are within the inertial subrange, we have 191 [19]

$$
\begin{gathered}
D\left(r_{c}, 0\right)=C_{E}\left(\epsilon r_{c}\right)^{2 / 3}, \\
D\left(0, \tau_{c}\right)=C_{L}\left(\epsilon \tau_{c}\right) .
\end{gathered}
$$

Inserting Eqs. (8) and (9) into Eq. (7) gives an expression for 195 the aspect ratios 
197

$$
r_{c}=V \tau_{c}
$$

198 where 199

$$
V=\left(C_{L} / C_{E}\right)^{2 / 3}\left(\epsilon \tau_{c}\right)^{1 / 2} \propto \tau_{c}^{1 / 2} .
$$

200 Therefore, the aspect ratio satisfies a power law in the iner201 tial range.

202 We now derive the scale-similarity model: for the cross203 ing point $\left(0, r_{c}\right)$ of the contour with the space separation axis 204 and any other points $(\tau, r)$ on the same contour, we have

$$
R(r, \tau)=R\left(r_{c}, 0\right) .
$$

206 The correlation function $R(r, \tau)$ can be expanded in a Taylor 207 power series about the origin $r=0$ and $\tau=0$ up to a second 208 order [12]

209

$$
\begin{aligned}
R(r, \tau)= & R(0,0)+\frac{\partial R}{\partial r}(0,0) r+\frac{\partial R}{\partial \tau}(0,0) \tau+\frac{1}{2} \frac{\partial^{2} R}{\partial r^{2}}(0,0) r^{2} \\
& +\frac{\partial^{2} R}{\partial r \partial \tau}(0,0) r \tau+\frac{1}{2} \frac{\partial^{2} R}{\partial \tau^{2}}(0,0) \tau^{2}+O\left(r^{3}, r^{2} \tau, r \tau^{2}, \tau^{3}\right) .
\end{aligned}
$$

210

211 The statistically isotropy and stationarity assumptions on the 212 Gaussian velocity fields imply [12]

213

$$
\frac{\partial R}{\partial r}(0,0)=\frac{\partial R}{\partial \tau}(0,0)=0, \frac{\partial^{2} R}{\partial r \partial \tau}(0,0)=0 .
$$

214 Thus, up to a second order approximation, the isocorrelation 215 contours $R(r, \tau)=C$ can be written in the form of

$$
r^{2}+V^{2} \tau^{2}=C^{\prime}
$$

217 where

$$
V^{2}=\frac{\partial^{2} R}{\partial \tau^{2}}(0,0)\left[\frac{\partial^{2} R}{\partial r^{2}}(0,0)\right]^{-1},
$$

219 and $C^{\prime}$ is a constant. Since both $C$ and $C^{\prime}$ denote the contour 220 levels, we don't distinguish between them without confusion. 221 Substitution of Eq. (15) into Eq. (12) yields

\section{2}

$$
r_{c}^{2}=r^{2}+(V \tau)^{2}
$$

223 Replacing of $r_{c}$ in Eq. (12) by Eq. (17) gives the scale224 similarity model

$$
R(r, \tau)=R\left(\sqrt{r^{2}+V^{2} \tau^{2}}, 0\right) .
$$

226 The Taylor series expansion is valid for small separations 227 and thus the model valid only for small separations. To ex228 tend this model to larger separations, we need to invoke the 229 scale-similarity assumption of LVCs with a second order ap230 proximation to the isocorrelation contours. Under the 231 second-order approximation, we can express the isocorrela232 tion contours by Eq. (15) with an aspect ratio $V=r_{c} / \tau_{c}$, that 233 is,

$$
r^{2}+V^{2} \tau^{2}=C
$$

235 where the aspect ratio $V$ is implicitly dependent on the sepa236 ration $r_{c}$ via the contour level $C$. The linear terms in Eq. (13) 237 vanishes due to the isotropy and stationarity assumptions on the Gaussian velocity fields, in which the contours have no 238 any preference direction. In terms of the scale-similarity as- 239 sumption, the aspect ratio can be determined by Eq. (11). 240 Taking the same procedure as the one from Eq. (15) to Eq. 241 (2), we obtain the scale-similarity model for the separations 242 in the inertial range.

The scale-similarity model suggests that a two-point and $\mathbf{2 4 4}$ two-time LVC can be obtained from an Eulerian velocity 245 correlation and a similarity transformation. The similarity 246 transformation is determined by the isocorrelation contours. 247 It converts between two pairs of space and time separations 248 at which the same correlation value is achieved. The Smith- 249 Hay model is essentially a linear approximation to the iso- 250 correlation contours. It is only valid for "frozen flows." In 251 this case, the particle pairs are convected by the frozen flows 252 which move at a uniform velocity $U$, without any distortion. 253 The convection velocity was mistaken to determine the La- 254 grangian decorrelation process. Some corrections to the con- 255 vection velocity were made to improve the Smith-Hay model 256 with some successes [18]. However, it remains to be a great 257 challenge to determine the convection velocity for the non- 258 frozen flows. The present model proposes a second approxi- 259 mation to the isocorrelation contours. It describes the La- 260 grangian decorrelation process in the "nonfrozen" flows 261 where the correlation functions decay as either space or time 262 separation increases. The dispersion velocity is defined as the 263 ratio of the spatial length scale to the temporal one. There- 264 fore, it measures the separation velocity of one particle rela- 265 tively to another fixed particle. The dependence of the dis- 266 persion velocity on the equivalent separation $r_{c}$ confirms that 267 particle dispersions rely on the instantaneous separations. 268 The larger dispersion velocity implies a faster decorrelation 269 process.

270

To derive the scale-similarity model for LVCs, we assume 271 that the isocorrelation contours have an approximately ellip- 272 tic shape. This approximation makes it possible to analyti- 273 cally derive the scale-similarity model. The present model is $\mathbf{2 7 4}$ a second approximation to the isocorrelation contours while 275 the Smith-Hay model is a first approximation to the ones. 276 Higher order approximations could be invoked to derive 277 more accurate expressions but they are not necessary for the $\mathbf{2 7 8}$ present purposes. The second order approximation is the $\mathbf{2 7 9}$ least order but most meaningful approximation to the corre- 280 lation functions.

281

It is ideal to apply the scale-similarity model to high- 282 Reynolds number turbulence with both space and time sepa- 283 rations in the inertial subrange. The inertial subrange be- 284 comes infinitely extensive in the limit of infinite Reynolds 285 number. However, in the practical case of finite Reynolds 286 numbers, the inertial subrange is finite [30]. Thus, the space 287 and time separations may belong to the different scaling sub- 288 ranges, such as the dissipation subrange and the inertial sub- 289 range. In this case, the scale-similarity model can still sur- 290 vive but the dispersion velocities have to be reformulated. 291 For example, if the temporal separation $\tau$ is in the inertial 292 range and the spatial separation $r$ in the dissipation range, we 293 then have Eq. (9) for the temporal separation $\tau_{c}$ and the 294 following equation for the spatial separation $r_{c}$ : 
296

$$
D\left(r_{c}, 0\right)=C_{E} \frac{\epsilon}{\nu}\left(r_{c}\right)^{2}
$$

297 Using the same procedure as the one from Eq. (7) to Eq. 298 (10), we have

$$
V=\left(C_{L} / C_{E}\right)^{1 / 2}\left(\frac{\nu}{\tau_{c}}\right)^{1 / 2}
$$

300

301

302 The dispersion velocity is the only one parameter in the 303 scale-similarity model. In this section, we will analytically 304 calculate the dispersion velocity from the Navier-Stokes 305 equations using the quasinormality assumption. The analyti306 cal results obtained will be used to study the Lagrangian 307 decorrelation process.

308 We recall the scale-similarity model of the form

309

$$
R(r, \tau)=R\left(\sqrt{r^{2}+V^{2} \tau^{2}}, 0\right) .
$$

310 Taking the second-order derivative of both sides of Eq. (22) 311 with respect to $\tau$ at $\tau=0$ and $r=r_{c}$, we obtain

312

$$
\frac{\partial^{2} R\left(r_{c}, 0\right)}{\partial \tau^{2}}=\frac{V^{2}(C)}{r_{c}} \frac{\partial R\left(r_{c}, 0\right)}{\partial r} .
$$

313 The left-hand side of Eq. (23) can be rewritten in Fourier 314 space and further simplified to

315

$$
\begin{aligned}
\frac{\partial^{2} R\left(r_{c}, 0\right)}{\partial \tau^{2}} & =\int_{-\infty}^{+\infty} \frac{\partial^{2} Q(k, 0)}{\partial \tau^{2}} \exp \left(-i \mathbf{k} \cdot \mathbf{r}_{c}\right) d^{3} \mathbf{k} \\
& =\int_{0}^{+\infty} \frac{\partial^{2} Q(k, 0)}{\partial \tau^{2}} \frac{\sin \left(k r_{c}\right)}{k r_{c}} 4 \pi k^{2} d k,
\end{aligned}
$$

316

317 where $Q(k, \tau)=\left\langle\hat{u}_{i}(\mathbf{k}, t) \hat{u}_{i}(-\mathbf{k}, t+\tau)\right\rangle$ is the time correlation of 318 velocity modes $\hat{u}_{i}(\mathbf{k}, t)$, seeing Eq. (2.26) on page 23 of the 319 classic book on DIA [10]. The time derivative of the mode 320 correlation can be evaluated using the Navier-Stokes equa321 tions and the quasinormality assumption, whose details can 322 be found from the derivation of Eq. (16b) in Ref. [31]. In the 323 evaluations, the time derivatives of velocity components in 324 the mode correlations can be calculated from the Navier325 Stokes equations, which yield the quadruple correlations. If 326 the velocity fields are quasinormal, the quadruple correla327 tions can be expressed by their pair correlations. It is shown 328 [32] that the quasinormality assumption is a reasonable ap329 proximation to calculate Eulerian and Lagrangian time cor330 relations in isotropic turbulence. This evaluation gives

331

$$
\frac{\partial^{2} Q(k, 0)}{\partial \tau^{2}}=-k Q(k, 0) \int_{0}^{+\infty} q E(q) j(q / k) d q
$$

332 where $E(k)=2 \pi k^{2} Q(k, 0)$ is the energy spectra and

333

$$
j(x)=\frac{\left(a^{2}-1\right)^{2} \log \frac{1+a}{1-a}-2 a+\frac{10}{3} a^{3}}{4 a^{4}},
$$

$$
a=\frac{2 x}{1+x^{2}}
$$

Submitting Eq. (25) into Eq. (24), we obtain

$$
\frac{\partial^{2} R\left(r_{c}, 0\right)}{\partial \tau^{2}}=-2 \int_{0}^{\infty} k E(k) d k \int_{0}^{\infty} q E(q) j(q / k) \frac{\sin \left(k r_{c}\right)}{k r_{c}} d q .
$$

The integrations in Eq. (27) are symmetric about $q$ and $k, 337$ and $j(q / k) \approx 16 q / 15 k$ for $q / k<1$. These considerations lead 338 to

$$
\begin{aligned}
\frac{\partial^{2} R\left(r_{c}, 0\right)}{\partial \tau^{2}} & =-4 \int_{0}^{\infty} k E(k) \int_{0}^{k} q E(q) j(q / k) \frac{\sin \left(k r_{c}\right)}{k r_{c}} d q d k \\
& =-\frac{64}{15} \int_{0}^{\infty} E(k) \frac{\sin \left(k r_{c}\right)}{k r_{c}} d k \int_{0}^{k} q^{2} E(q) d q .
\end{aligned}
$$

Inserting Eq. (28) into Eq. (23) gives an expression for the 342 dispersion velocity

$$
V^{2}=\frac{64}{15} \int_{0}^{\infty} E(k) \frac{\sin \left(k r_{c}\right)}{k r_{c}} d k \int_{0}^{k} q^{2} E(q) d q\left[\frac{R_{r}\left(r_{c}, 0\right)}{r_{c}}\right]^{-1} .
$$

The dispersion velocity $V$ depends on the spatial chord 345 length $r_{c}$. The spatial chord length $r_{c}$ represents the charac- 346 teristic length scale of the isocorrelation contour $R(r, \tau)=C, 347$ while the temporal chord length $\tau_{c}$ represents the character- 348 istic time scale of the isocorrelation contour $R(r, \tau)=C$ such 349 that $R\left(0, \tau_{c}\right)=C$. Therefore, the dispersion velocity is a local 350 quantity. However, the sweeping velocity in Eulerian time 351 correlations is a global one. Note that the sweeping velocity 352 for the two-point and two-time Eulerian velocity correlations 353 is given by $[11,16]$

354

$$
U^{2}=\int_{0}^{+\infty} E(k) d k
$$

If the characteristic length scale $r_{c}$ is very small, for example 356 $r_{c} \rightarrow 0$, then $\sin \left(k r_{c}\right) / k r_{c} \rightarrow 1$ and $R_{r}\left(r_{c}, 0\right) / r_{c} \rightarrow R_{r r}(0,0) 357$ $=\frac{2}{3} \int_{0}^{+\infty} k^{2} E(k) d k$. The dispersion velocity $V$ can be thus sim- 358 plified to

$$
V^{2}=\int_{0}^{+\infty} E(k) d k \int_{0}^{k} q^{2} E(q) d q\left[\int_{0}^{+\infty} k^{2} E(k)\right]^{-1} .
$$

Therefore, the dispersion velocity is determined by the en- 361 strophy $\int_{0}^{k} q^{2} E(q) d q$ in additional to the energy spectra $E(k) .362$

Comparing Eq. (30) with Eq. (31) gives

363

$$
\int_{0}^{+\infty} k^{2} E(k) d k\left[U^{2}-V^{2}\right]=\int_{0}^{+\infty} E(k) d k \int_{k}^{+\infty} q^{2} E(q) d q>0 .
$$

This implies that the Lagrangian dispersion velocity is 365 smaller than the Eulerian sweeping velocity. Therefore, the 366 LVCs decay more slowly than the Eulerian velocity correla- 367 tions in time. This result is consistent with the one obtained 368 
TABLE I. Relevant parameters and statistic quantities in the DNS.

\begin{tabular}{lcc}
\hline \hline Simulation grid & $128^{3}$ & $256^{3}$ \\
\hline $\operatorname{Re}_{\lambda}$ & 66.4 & 102.05 \\
$u^{\prime}$ & 18.73 & 18.59 \\
$L_{f}$ & 1.925 & 1.819 \\
$\lambda$ & 0.4358 & 0.2675 \\
$\eta$ & 0.0272 & 0.0135 \\
$\mathrm{CFL}$ & 0.56 & 0.3150 \\
$k_{\max } \eta$ & 1.158 & 1.1479 \\
\hline \hline
\end{tabular}

369 from the LHDIA [23], the dimensional analysis [33] and the 370 Taylor series expansion [31].

371 An extension of the scale-similarity model to turbulent 372 shear flows is possible. Here, we only present a formal ex373 tension to homogeneous sheared turbulence. In this case, the 374 isocorrelation contours remain elliptic in shape. Therefore, 375 the scale-similarity model has the same expression as the one 376 for isotropic turbulence. The dispersion velocity in the model 377 can be formally calculated, which is given by

$$
V^{2}=\frac{\int_{0}^{+\infty}\left[\frac{1}{3} S^{2}+\frac{32}{15} \int_{0}^{k} q^{2} E(q) d q\right] \frac{\sin \left(k r_{c}\right)}{k r_{c}} E(k) d k}{\frac{1}{r_{c}^{2}} \int_{0}^{+\infty}\left[\frac{\sin \left(k r_{c}\right)}{k r_{c}}-\cos \left(k r_{c}\right)\right] d k},
$$

378

379 where $S$ is a shear rate associated with the mean velocity: $380 U_{1}=S y, U_{2}=0$, and $U_{3}=0$. It can be found from Eq. (33) that 381 the shear increases the dispersion velocity and thus enhances 382 the Lagrangian decorrelation process. Further studies are 383 needed to validate the scale-similarity model in turbulent 384 shear flows.

\section{IV. NUMERICAL VERIFICATION OF THE SCALE- 386 SIMILARITY MODEL}

387 The DNS for isotropic turbulence was performed using a 388 pseudospectral method. The computational domain of the 389 three-dimensional Navier-Stokes equations is a box of each 390 side length $L=2 \pi$, where the periodic boundary conditions 391 are applied. To maintain the turbulence stationary, an exter392 nal force $f(k)$ is imposed on the first two shells of wavenum-
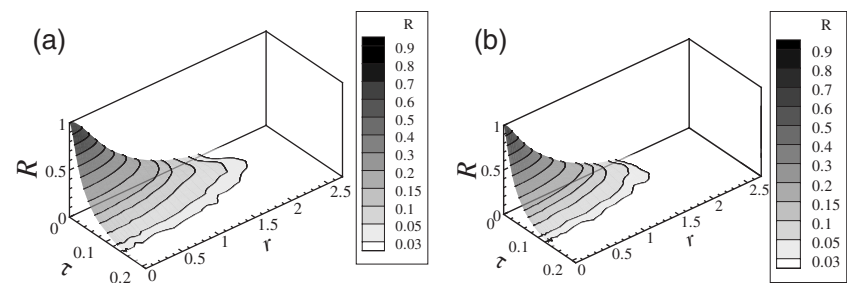

FIG. 1. The surfaces of the Lagrangian velocity correlations as functions of space and time separations at two grids: (a) $128^{3}$ and (b) $256^{3}$.
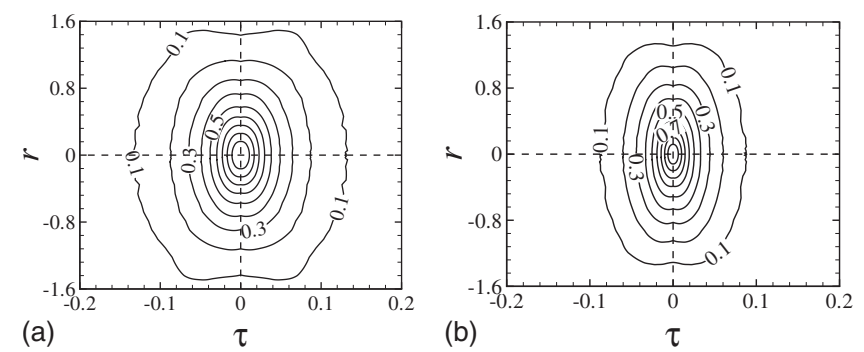

FIG. 2. The isocorrelation contours at two grids: (a) $128^{3}$ and (b) $256^{3}$.

bers $k=1,2$. Aliasing errors are removed through the two- 393 thirds truncation rule. The Adams-Bashforth scheme is used 394 for time advance. Two cases are run in the present study: 395 case 1 on a $128^{3}$ grid and case 2 on a $256^{3}$ grid, respectively. 396 The relevant parameters and statistic quantities in the DNS 397 are listed in Table I, where $\operatorname{Re}_{\lambda}$ is the Taylor-microscale Rey- 398 nolds number, $u^{\prime}$ the root-mean-square of velocity fluctua- 399 tions, $L_{f}$ the integral length scale, $\lambda$ the Taylor microscale 400 and $\eta$ the Kolmogorov length scale. CFL denotes the CFL $401 \mathrm{AQ}$ : number. $k_{\max } \eta$ describes the spatial resolution. 402

Figure 1 plots the surface of the Lagrangian velocity cor- 403 relations obtained from the DNS at two grids $128^{3}$ and $256^{3} .404$ The correlation curves decay faster in the direction of time 405 separation axis than the ones in the direction of space sepa- 406 ration axis. The surfaces look like paraboloidal in shape. 407

Figure 2 presents the isocorrelation contours at different 408 levels. They are very different from the straight lines as im- 409 plied by the Smith-Hay model. They look more like elliptic 410 curves, which supports the assumption on the present scale- 411 similarity model. It is clearly observed that the contours have 412 no any preference direction: their principal axes are coinci- 413 dent with the space and time separation axes respectively. 414 This is different from the Eulerian space-time correlations in 415 turbulent shear flows, where the principal axes are not 416 aligned with either of space and time axes [34,35].

417

Figure 3 shows the lengths of major axes of the isocorre- 418 lation contours as a function of the lengths of their minor 419 axes. The lengths of the axes can be directly calculated from 420 the DNS data: the length of a major axis is the largest dis- 421 tance between two points on the isocorrelation contours and 422 the length of a minor axis is the shortest distance between 423 them. In the present case, the major axis is the space sepa- 424 ration one and the minor axis is the time separation one. It 425 can be observed that there exist two scaling subranges: $r_{c} 426$
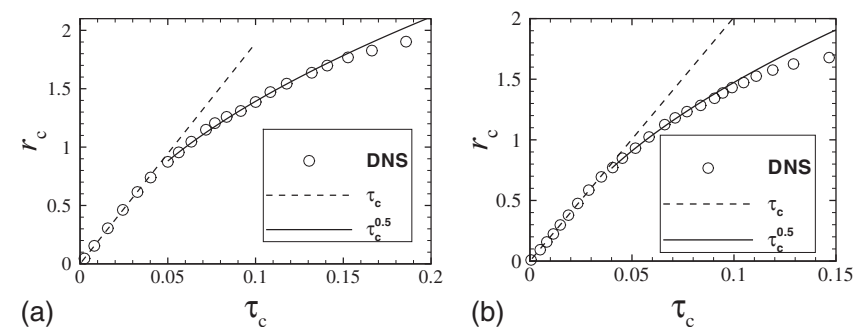

FIG. 3. The lengths of the major axes of the isocorrelation contours as functions of the lengths of the minor axes at two grids: (a) $128^{3}$ and (b) $256^{3}$. 


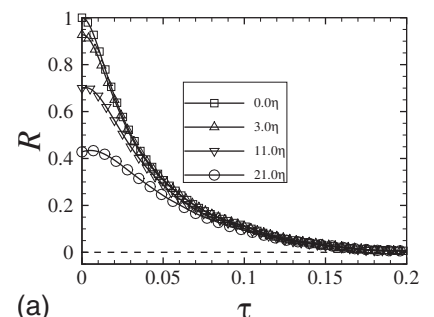

(a)

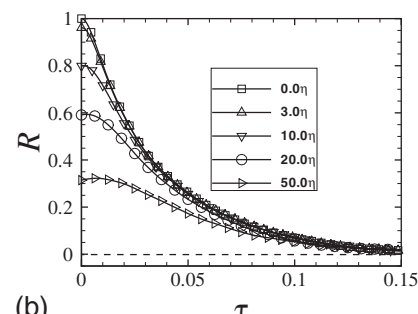

(b)

FIG. 4. The Lagrangian velocity correlations for different space separations at two grids: (a) $128^{3}$ and (b) $256^{3}$.

$427 \propto \tau_{c}$ for both $r_{c}$ and $\tau_{c}$ in the dissipation subrange and $r_{c}$ $428 \propto \tau_{c}^{0.5}$ for $r_{c}$ in the dissipation subrange and $\tau_{c}$ in the inertial 429 subrange. The scaling ranges for the large Reynolds number $430(\mathrm{Re}=102.05)$ are more evident than the one for the small one $431(\mathrm{Re}=66.40)$. This observation supports the assumption that 432 the aspect ratios of the elliptic contours satisfy the scaling 433 laws.

434 Figure 4 shows the evolution of the LVCs with time sepa435 ration for different initial separations. Those correlation 436 curves monotonously decrease for initially smaller separa437 tions while they initially increase and then decrease for ini438 tially larger separations. The scale-similarity model predicts 439 an universal form for the LVCs: $R(r, \tau)=R\left(\sqrt{r^{2}+V^{2} \tau^{2}}, 0\right)$. If 440 the LVCs are plotted against the rescaled separations $r^{*}$ $441=\sqrt{r^{2}+V^{2} \tau^{2}}$, the curves $R$ versus $r^{*}$ for different separations $r$ 442 should collapse into the universal form $R\left(r^{*}, 0\right)$. Figure 5 443 plots those curves together against the rescaled separation 444 axis defined by $r^{*}=\sqrt{r^{2}+V^{2} \tau^{2}}$. The dispersion velocity $V$ here 445 is calculated from the DNS data, which is the aspect ratio of 446 the isocorrelation contours. Evidently, those curves collapse 447 to a single curve. This collapse supports the scale-similarity 448 model.

\section{DISCUSSION AND CONCLUSIONS}

450 In summary, we develop a scale-similarity model for 451 LVCs in isotropic turbulence

452

$$
R(r, \tau)=R\left(\sqrt{r^{2}+V^{2} \tau^{2}}, 0\right)
$$

453 which relates the Lagrangian velocity correlations $R(r, \tau)$ to 454 the space correlations $R(r, 0)$. The dispersion velocity can be 455 obtained from the following two equations

456

$$
V^{2}=\frac{64}{15} \int_{0}^{\infty}\left[E(k) \frac{\sin \left(k r_{c}\right)}{k r_{c}} \int_{0}^{k} q^{2} E(q) d q\right]
$$

457

$$
\times d k\left[-\frac{1}{r_{c}} \frac{\partial R\left(r_{c}, 0\right)}{\partial r}\right]^{-1},
$$

458

$$
r_{c}^{2}=r^{2}+V^{2} \tau^{2}
$$

459 This model suggests an interpretation of the Lagrangian 460 decorrelation process: a fluid element is distorted by the ed461 dies of the sizes comparable with the fluid element. The dis462 persion velocity measures the particle separations induced by
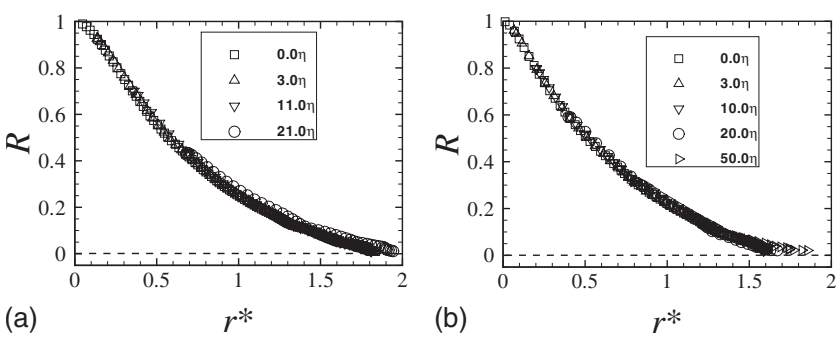

FIG. 5. The Lagrangian velocity correlations for different space separations are plotted against the rescaled separation $r^{*}$ $=\sqrt{r^{2}+(V \tau)^{2}}$ at two grids: (a) $128^{3}$ and (b) $256^{3}$.

the distortion of the fluid element. The Lagrangian decorre- 463 lation process is very different from the Eulerian decorrela- 464 tion process, in which small eddies are swept by the energy- 465 containing eddies and the sweeping velocity dominates this 466 decorrelation process. An analytical calculation shows that 467 the dispersion velocity is smaller than the sweeping one. 468 Therefore, the Lagrangian decorrelation process is more 469 slowly than the Eulerian decorrelation one.

470

The scale-similarity model is a second approximation to 471 the isocontours of Lagrangian velocity correlations, while 472 the Hay-Smith model is a first approximation to the ones. 473 The isocorrelation contours offer a transformation between 474 the Lagrangian and Eulerian velocity correlations. As a con- 475 sequence of the classic Kolmogorov similarity hypothesis, 476 the transformation should be scale similar. The DNS of iso- 477 tropic turbulence supports the scale-similarity model: the $\mathbf{4 7 8}$ correlation functions exhibit a fair good collapse, when plot- 479 ted against the normalized space and time separations de- 480 fined by the model.

481

The scale-similarity model implies that Lagrangian veloc- 482 ity correlations are mainly determined by the energy spectra 483 and the dispersion velocities. Since the dispersion velocities 484 are mainly determined by enstrophy spectra in addition to the $\mathbf{4 8 5}$ energy spectra, one can conclude that an accurate prediction 486 of LES on the energy spectra may not ensure the accurate 487 prediction of Lagrangian velocity correlations. The typical 488 class of eddy viscosity SGS models is constructively based 489 on the energy balance equations. Therefore, it many not cor- 490 rectly predict the Lagrangian velocity correlations. The criti- 491 cal comparison between the Lagrangian velocity correlations 492 in DNS and the ones in LES supports this conclusion [14]. 493 Therefore, the present model may offer a clue to develop the 494 SGS modeling for LES of particle-laden turbulence.

495

\section{ACKNOWLEDGMENTS}

496

This work was supported by Chinese Academy of Sci- 497 ences under the Innovative Project "Multiscale modeling and 498 simulation in complex systems" (Grant No. KJCX-SW-L08), 499 National Basic Research Program of China (973 Program) 500 under Project No. 2007CB814800 and National Natural Sci- 501 ence Foundation of China under Projects No. 10325211, No. 502 10628206, No. 10732090, and No. 10702074. G.J. acknowl- 503 edges the LNM special funding for young investigators. 
505 [1] L. F. Richardson, Proc. R. Soc. London, Ser. A 110, 709 506 (1926).

507 [2] B. L. Sawford, Annu. Rev. Fluid Mech. 33, 289 (2001).

508 [3] P. K. Yeung, Annu. Rev. Fluid Mech. 34, 115 (2002).

509 [4] G. I. Taylor, Proc. London Math. Soc. s2-20, 196 (1922).

510 [5] G. K. Batchelor, Q. J. R. Meteorol. Soc. 76, 133 (1950).

511 [6] G. K. Batchelor, Proc. Cambridge Philos. Soc. 48, 345 (1952).

512 [7] L. I. Zaichik and V. M. Alipchenkov, Phys. Fluids 15, 1776 513 (2003)

514 [8] G.-W. He and Z.-F. Zhang, Phys. Rev. E 70, 036309 (2004).

515 [9] M. W. Reeks, J. Fluid Mech. 522, 263 (2005).

516 [10] D. C. Leslie, Modern Development in the Theory of Turbu517 lence (Oxford University Press, London, 1972).

518 [11] R. H. Kraichnan, Phys. Fluids 7, 1723 (1964).

519 [12] T. Gotoh, R. S. Rogallo, J. R. Herring, and R. H. Kraichnan, 520 Phys. Fluids A 5, 2846 (1993).

521 [13] R. Rubinstein and Y. Zhou, Phys. Fluids 11, 2288 (1999).

522 [14] Y. Yang, G.-W. He, and L.-P. Wang, J. Turbul. 9, 1 (2008).

523 [15] G.-W. He, R. Rubinstein, and L.-P. Wang, Phys. Fluids 14, 5242186 (2002).

525 [16] G.-W. He, M. Wang, and S.-K. Lele, Phys. Fluids 16, 3859 526 (2004).

527 [17] F. B. Smith and J. S. Hay, Q. J. R. Meteorol. Soc. 87, 82 $528 \quad(1961)$.

529 [18] B. L. Sawford, Q. J. R. Meteorol. Soc. 108, 191 (1982).

530 [19] A. S. Monin and A. M. Yaglom, Statistical Fluid Mechanics, edited by J. Lumley (MIT Press, Cambridge, MA, 1975), Vols. 531 1 and 2.

532

[20] R. H. Kraichnan, Phys. Fluids 9, 1728 (1966). 533

[21] S. Corrsin, Proceeding of the Oxford Symposium on Atmo- 534 spheric Diffusion and Air Pollution (Academic, New York, 535 1960), p. 162.

[22] R. H. Kraichnan, J. Fluid Mech. 81, 385 (1977). 537

[23] R. H. Kraichnan, Phys. Fluids 8, 575 (1965). 538

[24] Y. Kaneda, Fluid Dyn. Res. 39, 526 (2007). 539

[25] T. Gotoh and Y. Kaneda, Phys. Fluids A 3, 2426 (1991). 540

[26] P. A. Durbin, J. Fluid Mech. 100, 279 (1980). 541

[27] M. S. Borgas and B. L. Sawford, J. Fluid Mech. 279, 69542 (1994).

543

[28] D. J. Thomson, Atmos. Environ. 25A, 1725 (1991). 544

[29] M. Bourgoin, N. T. Ouellette, H. T. Xu, J. Berg, and E. Boden- 545 schatz, Science 311, 835 (2006).

546

[30] G.-W. He, S.-Y. Chen, R. H. Kraichnan, R. Zhang, and Y. 547 Zhou, Phys. Rev. Lett. 81, 4636 (1998). 548

[31] Y. Kaneda, Phys. Fluids A 5, 2835 (1993). 549

[32] Y. Kaneda, T. Ishihara, and G. Koji, Phys. Fluids 11, 2154550 (1999).

551

[33] H. Tennekes, J. Fluid Mech. 67, 561 (1975). 552

[34] G.-W. He and J.-B. Zhang, Phys. Rev. E 73, 055303(R) 553 (2006).

554

[35] X. Zhao and G.-W. He, Phys. Rev. E 79, 046316 (2009). 555 